\title{
Identifying the nutritional profile of outpatient haemodialysis patients in Beaumont Hospital
}

\section{Abstract}

Beaumont Hospital Kidney Centre, Dublin is currently the largest provider of haemodialysis in Ireland. There is a dedicated renal dietetic team to address the nutritional needs of these patients. In order to provide an optimal service, a thorough understanding and insight of the service demands is needed. Currently there is no nutritional profile of these patients. Similarly the Renal dietetic team have not been in a position to identify if they are meeting current best practice guidelines, as outline by the National Kidney Foundation. E-Med, an online renal electronic notes system, was used to provide all dietetic notes during 2018. Using an audit template, an audit of these notes for outpatient haemodialysis patients was carried out to identify a number of nutritional issues. $75 \%$ $(123 / 163)$ of outpatient haemodialysis patients received direct dietetic intervention in $2018.76 \%$ of these patients demonstrated more than one nutritional issue and $16 \%$ displayed more than five nutritional issues. The most prevalent nutritional issues arising were hyperkalemia $(41 \%)$ and hyperphosphatemia (33\%). Currently guidelines, which recommend a dietetic review, every 3-4 months are not being met. $53 \%$ of patients received either none or only one direct dietetic intervention in this time period. The recommended ratio of 1:100 (dietitian: haemodialysis patient) is also not being met. Currently the ratio stands at 1:243 as the dietitian responsible for haemodialysis patients is also responsible for peritoneal dialysis patients. The average length of time recorded for each direct intervention was 1 hour. Beaumont Hospital haemodialysis outpatient cohort is a nutritionally complex group of patients, which require ongoing and frequent dietetic input. Beaumont Hospital renal dietitians are successfully providing at least one episode of direct intervention to the majority $(75 \%)$ of the outpatient haemodialysis patients, suggesting efficient and effective use of available resources. An increase in dietetic staffing for the renal service could offer the potential to provide direct dietetic input to all haemodialysis patients within the hospital and to also provide an increased number of reviews to patients, as per guidelines. It is possible that the amount of time spent on dietetic patient assessments is being under-reported and further work is needed to cross-reference these figures using an alternative statistical package.

\section{Conflict of Interest}

There is no conflict of interest 